\title{
Optimizing Protective Clothing Design for Hot Outdoor Environments
}

\author{
Uwe Reischl ${ }^{1, a}$, Gang Sun ${ }^{2, b}$ \\ Qazaleh Bahramian ${ }^{2, c}$, Zhuqing Xue ${ }^{1, d}$ \\ ${ }^{1}$ Boise State University, Boise, Idaho USA, ${ }^{2}$ University of California, Davis, California USA \\ aureischl@boisestate.edu, bgysun@ucdavis.edu, cqbahramian@ucdavis.edu, \\ dzhuqingxue@u.boisestate.edu
}

\section{Keywords: Protective Clothing, Heat Radiation, Thermal Cross-Over Points}

\begin{abstract}
Thermal manikin tests were performed on selected protective garments to assess their heat transfer properties during exposure to infrared heat radiation. Comparing a newly developed Infrared (IR) Heat Radiation attenuatuion vest with a standard winter jacket, it could be seen that that the IR Vest provided a thermal comfort benefit above a heat radiation exposure level of 150 Watts while the winter jacket provided a thermal benefit above a 450 Watt heat radiation exposure level. The study identified important temperature cross-over points which define the thermodynamic properties and also characterize the heat radiation attenuation and heat insulation properties of garments. The evaluation method is seen as a new design tool for optimizing garment comfort and safety.
\end{abstract}

\section{Introduction}

Innovative use of clothing materials and creative approaches to garment design can help reduce clothing heat stress during exposure to hot outdoor environments. While garments can offer a barrier to solar heat radiation, the garments also create heat stress by limiting the dissipation of metabolic heat. The goal of this study was to identify an evaluation method suitable for optimizing garment design balancing garment insulation with garment heat radiation attenuation. Experiments were conducted to document the thermodynamic characteristics of selected garments under controlled exposures conditions to determine the infrared heat radiation penetration, fabric radiation absorption, and garment heat insulation.

\section{Background}

Agricultural workers and construction workers exposed to solar heat radiation in hot and humid climates are at high risk of suffering heat related disorders. Use of tents, hats, and other protective equipment are sometimes used to reduce the solar heat exposure. However, such measures are often impractical when employees are required to change their working postures or must move frequently to new locations. Attempting to reduce heat stress imposed by solar heat radiation using protective clothing poses a significant ergonomic and public health challenge.

Physiologically, the human body is a metabolic heat generating system which must maintain a balance between heat loss and heat gain within a narrow range of body temperature. Environmental parameters such as air temperature, air velocity, radiant heat, and humidity can affect this delicate balance $[1,2,3]$. Clothing material and garment design can influence the heat balance by promoting or reducing heat exchange through sweat evaporation, convection, conduction, and heat radiation [4]. The protective performance of fabrics under such conditions is related to the chemical and physical structure of the material including thickness and weight. It has been shown that woven textile materials do not offer a good barrier against infrared radiation. However, the performance is better when the fabric thickness is greater and when the fabric material is heavier [5]. 


\section{Thermal Manikin}

An inflatable thermal mannequin system illustrated in Fig. 1 was used for this study. The manikin has the shape of a standing adult male and is heated at a constant temperature and inflated at a constant pressure. The mannequin's dimensions corresponded to a "Medium" sized person. Heat loss and heat gain is determined by the difference between the temperature of mannequin input air and the temperature of the mannequin output air, i.e., $\Delta \mathrm{T}^{0} \mathrm{C}$. The system is calibrated and allows for a conversion from the $\Delta \mathrm{T}^{0} \mathrm{C}$ value to power units, i.e., Watts. This value is used as the dependent variable in the experiments. Clothing and heat radiation exposure conditions are designated as independent variables. A semi-nude configuration provides a data reference and a "control" comparison. Fig. 2 illustrates the semi-nude "control" configuration, the IR Vest configuration, and the jacket configuration. These three configurations were used in this study.

The inflatable thermal mannequin represents a technology platform which allows a garment system to be evaluated accurately for convective, conductive, and radiative heat exchange. While the mannequin does not simulate human physiological responses, the system provides a stable and accurate platform for determining the standard thermodynamic properties of a wide range of garment configurations.

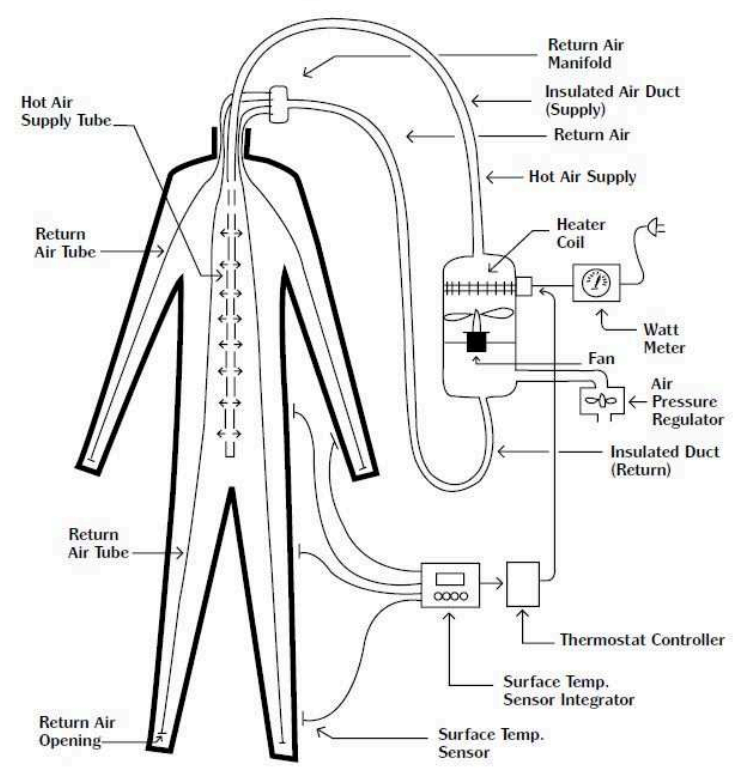

Fig.1. Illustration of the thermal manikin system used in evaluating the heat transfer dynamics of protective clothing.

\section{Procedures}

Thermal manikin tests were conducted inside a temperature controlled laboratory using previously described protocols [6]. Clothing configurations that were evaluated and compared to the seminude configuration included a prototype infrared reflecting vest (IR Vest) and a standard winter jacket. The semi-nude configuration, the IR Vest, and the winter jacket are illustrated in Fig. 2. The two garments were selected because of their performance differences in attenuating infrared heat radiation and their differences in heat insulation. Infrared heat radiation exposures were always preceded by the semi-nude "control" configuration. Infrared heat radiation intensity levels ranged from 0 Watts to 500 Watts in 100 Watt increments. 


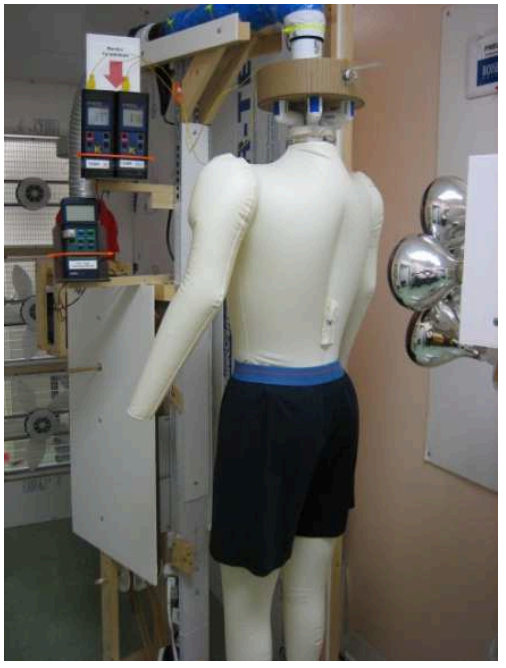

A

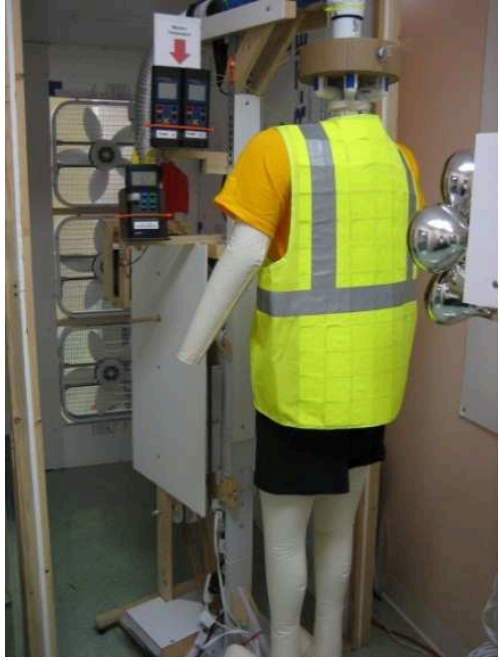

B

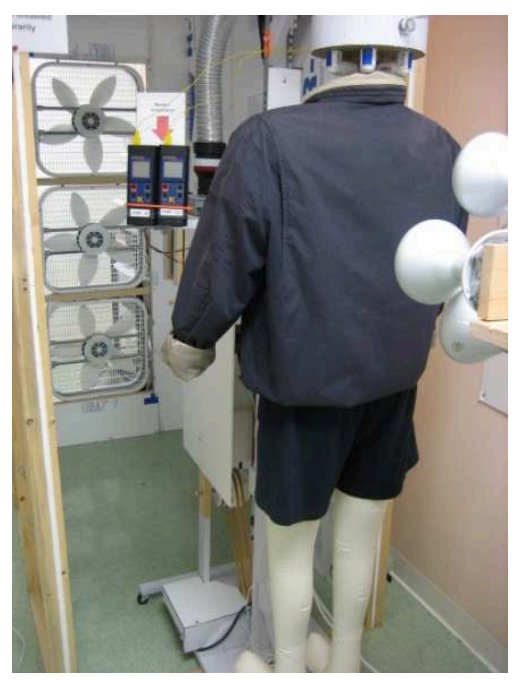

$\mathrm{C}$

Fig.2. Illustration of the thermal manikin in a semi-nude "control" configuration (A), wearing an IR Vest (B), and wearing a winter jacket (C).

\section{Results and Analysis}

Exposure of the semi-nude manikin to heat radiation up to 500 Watts resulted in a linear increase in the manikin's body temperature. When the manikin was clothed with the IR Vest and then exposed to the same heat radiation levels, a linear rise in the manikin temperature was also observed. However, the slope of this increase was lower than the slope observed for the semi-nude manikin. When the manikin was clothed with the winter jacket, the slope was even smaller. The slopes observed for the IR Vest and the winter Jacket describe the heat radiation attenuation characteristic of the garment, i.e., the lower the slope, the higher the heat radiation attenuation. However, the insulation heat gain observed for the winter Jacket was higher than the insulation heat gain observed for the IR Vest. No insulation heat gain was observed for the semi-nude manikin.

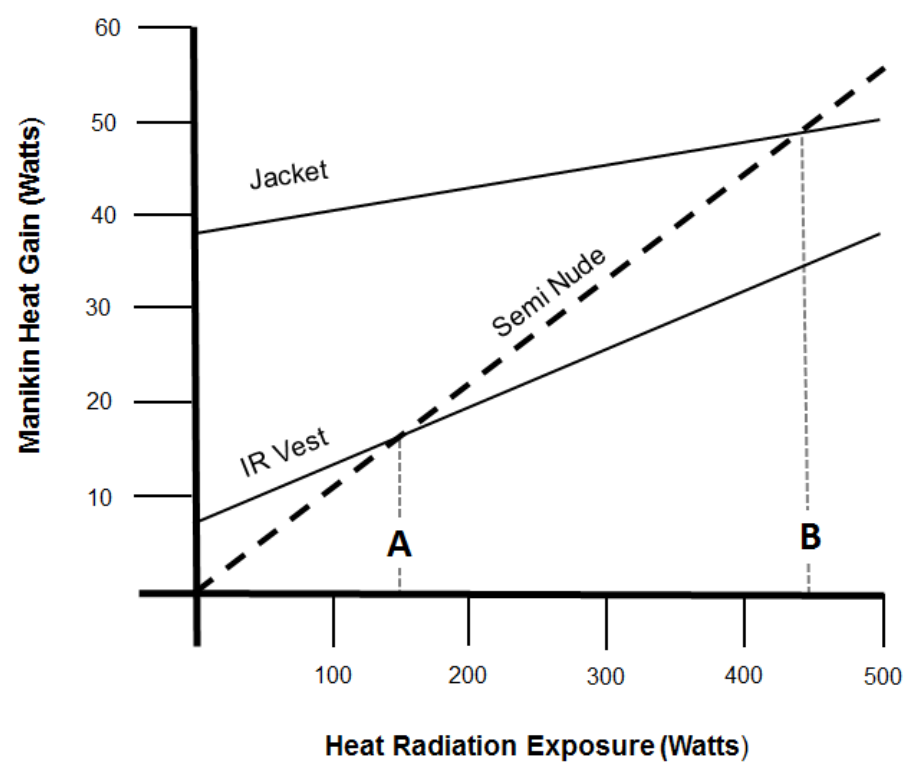

Fig. 3. Illustration of manikin heat gain associated with the semi-nude, IR Vest, and winter Jacket configurations during exposure to increasing infrared heat radiation levels. 
Fig. 3 illustrates the relationship between manikin heat gain, heat radiation exposure, and clothing. It can be seen that the semi-nude manikin gained the most heat when exposed to the heat radiation but exhibited no heat gain due to clothing insulation. The winter jacket exhibited the lowest heat gain due to heat radiation but exhibited the highest heat gain due to garment insulation. The IR Vest exhibited values between the two.

Total heat loading (heat gain) observed for the manikin wearing garments included two contributors: Heat absorbed from the infrared radiation that penetrated the garments and heat retention due to clothing insulation. To determine which garment performed the best, the graphics in Fig. 3 can be consulted. The IR Vest intersects the "control" graph at point "A" while the winter jacket graph intersects the "control" graph at point "B". These two "cross-over" points define the conditions where the radiation heat load imposed on the semi-nude manikin is equivalent to the heat load imposed on the manikin by both heat radiation and the garment insulation. Garments with a "low" cross-over point are superior over garments with a "high" cross-over point since the garments with a low cross-over point impose lower insulation heat stress while providing better infrared radiation attenuation.

\section{Conclusions}

The study identified temperature cross-over points which are able to define the thermodynamic properties of protective garments and characterizes the heat radiation attenuation and heat insulation properties of the garments. The evaluation method can be seen as a tool for designing garments. The thermal cross-over point offers a new approach to the improvement of garment comfort and safety. The thermal manikin system used in this study offers the necessary technology platform for such work.

\section{References}

[1] P. Bishop, G. Smith, P. Ray, J. Beaird, J. Smith, J. (1994). Empirical Prediction of Physiological Response to Prolonged Work in Encapsulating Protective Clothing, Ergonomics 37, (1994) 1503-1540.

[2] S. Montain, M. Sawka, B. Cadarette, M. Quigley, J. Mckay, Physiological Tolerance to Uncompensable Heat Stress: Effects of Exercise Intensity, Protective Clothing, and Climate, J. Appl. Physiol. 77, (1994) 216-222.

[3] I. Holmer, Protective Clothing and Heat Stress, Ergonomics 38, (1995) 166-182.

[4] U. Reischl, C. Colby, "PNEUMOTECH": A New Tool for Evaluating the Thermal Characteristics of Clothing Systems. Proceedings of the $4^{\text {th }}$ International Ergonomics Conference, In B. Mijovic, Editor. (2010). 29-36, Zagreb, Croatia.

[5] G.Y. Sun, X.S. Zhang, N. Pan, Radiant Protective and Transport Properties of Fabrics used by Wildland Firefighters. Textile Research Journal, 70 (2000) 567-573.

[6] B. Mijovic, Z. Skenderi, J. Camara, U. Reischl, C. Colby, A. Mermerian, Inflatable Mannequin for Testing Thermal Properties of Clothing. Proceedings of the $17^{\text {th }}$ World Congress on Ergonomics. Beijing, IEA. (2009). 